Rabaska

Revue d'ethnologie de l'Amérique française

\title{
Programme de français/Program in French Studies (Université de New-York à Albany)
}

\section{Cynthia Fox}

Volume 10, 2012

URI : https://id.erudit.org/iderudit/1013591ar

DOI : https://doi.org/10.7202/1013591ar

Aller au sommaire du numéro

Éditeur(s)

Société québécoise d'ethnologie

ISSN

1703-7433 (imprimé)

1916-7350 (numérique)

Découvrir la revue

Citer ce document

Fox, C. (2012). Programme de français/Program in French Studies (Université de New-York à Albany). Rabaska, 10, 344-345. https://doi.org/10.7202/1013591ar d'utilisation que vous pouvez consulter en ligne.

https://apropos.erudit.org/fr/usagers/politique-dutilisation/ 


\section{ÉTATS-UNIS}

\section{Programme de français/Program in French Studies}

Department of Languages, Literatures and Cultures

Humanities 235

University at Albany, State University of New-York

1400 Washington Avenue Téléphone : (518) 442-4102

Albany, NY 12222

Courriel : cfox@albany.edu

LE PATRIMOINE FRANÇAIS DE L'ÉTAT DE NEW-YorK

\section{A. J'aime New-York}

Fruit des recherches d'une équipe franco-américaine, J'aime New-York, guide bilingue du patrimoine français de l'État de New-York, a été publié à la State University of New-York (SunY) Press en 2012 sous la direction d'Éloïse A. Brière. Le livre de 113 pages permet de découvrir des lieux de l'État qui témoignent de la présence française de diverses origines : Canada, Europe, Caraïbe. Organisé par régions, le guide illustre les liens qui unissent New-York et la France dès 1523, et New-York et le Canada dès 1609, lorsque Samuel de Champlain découvrit le lac qui porte son nom. Les nombreux sites décrits dans le guide, comme les «Petits Canada » de Cohoes et de la ville de New-York ainsi que les établissements fondés par les Français qui fuyaient des persécutions religieuses ou politiques, méritent tous le détour. Disponible chez Suny Press : suny@presswarehouse.com.

\section{B. Le français parlé dans la région de Plattsburgh}

En mai, 2011, Gregg Castellucci a soutenu une thèse de premier cycle sous la direction de Cynthia Fox portant sur le français parlé dans le comté de Clinton dans le nord-est de l'État de New-York. La langue y est présente depuis 1763 et se parle encore à la maison aujourd'hui par une minorité non négligeable qui monte jusqu'à 7,6\% de la population selon la localité d'après le recensement de 2000. Cependant, la variété n'a pas été étudiée auparavant. La thèse, A description of the Plattsburgh, NY dialect of North American French and an analysis of its liaison retention and innovation, est basée sur un corpus d'entretiens sociolinguistiques faits entre septembre 2010 et janvier 2011 auprès de trente et un locuteurs habitant soit la ville de Plattsburgh, soit les villages de Beekmantown, Chazy, Peru et Champlain qui se trouvent dans la partie est du comté aux environs de Plattsburgh. Les locuteurs sont répartis selon le sexe (16 hommes et 15 femmes) et l'âge (de 55 à 89 ans). La majorité ( $n=20)$ est née ou arrivée dans la région avant l'âge de dix ans et constitue le groupe «population originaire ». Le groupe « population intégrée » $(n=11)$ comprend les locuteurs qui sont arrivés de Québec ou d'autres régions francophones après l'âge de 10 ans, mais qui ont passé au moins 40 ans dans la région. Cette division permet de distinguer les traits linguistiques indigènes de ceux qui ont été importés plus récemment. Les entretiens durent entre trente minutes et trois heures. De nature descriptive, la thèse énumère plusieurs traits qui suggèrent que le « français de Plattsburgh » se distin- 
gue du français franco-américain parlé dans d'autres communautés du nord-est des États-Unis et représente donc une sous-variété distincte du français québécois. Elle a obtenu la mention honorable au concours du Prix du Prévôt pour les recherches du premier cycle.

LE PROJET « LE FRANÇAIS FRANCO-AMÉRICAIN 》

L'analyse des données du corpus sociolinguistique du français parlé en NouvelleAngleterre constitué par C. Fox et sa collaboratrice Jane Smith de l'Université du Maine (cf. Rabaska, vol. 6, p. 277-278) continue bon train.

C. Fox est également collaboratrice au projet de recherches Le français à la mesure d'un continent : un patrimoine en partage, subventionné par le Conseil de recherches en sciences humaines du Canada et dirigé par France Martineau de l'Université d'Ottawa. Le corpus Fox-Smith sera éventuellement incorporé au Corpus des français d'Amérique du Nord, un ensemble unifié de documents textuels et oraux qui permettra de brosser l'histoire linguistique et culturelle des francophones du Canada et des États-Unis, ce qui est l'un des objectifs de ce grand projet concerté.

CyNTHIA Fox 